\title{
Fatores associados ao uso de serviços odontológicos públicos por adultos no estado de São Paulo, Brasil, 2016
}

\author{
Factors associated with public dental service use by adults in the \\ state of São Paulo, Brazil, 2016
}

Suelen Garcia Oliveira da Fonseca (http://orcid.org/0000-0002-0512-7110) ${ }^{1}$

Emílio Prado da Fonseca (http://orcid.org/0000-0002-6810-133X) ${ }^{1}$

Marcelo de Castro Meneghim (https://orcid.org/0000-0003-2673-3627) ${ }^{1}$

${ }^{1}$ Faculdade de Odontologia

\begin{abstract}
The aim of this study was to investigate the factors associated with public dental care use by adults in the State of São Paulo, Brazil. A cross-sectional study was conducted with a representative sample of adults aged 35 to 44 years using data from the 2015 Epidemiological Survey of the Oral Health Status of the Population of the State of São Paulo (SBSP-2015). Multivariate logistic regression was performed using variables based on a model proposed by Andersen for predicting access to public dental services. Results: 3,421 (59.9\%) adults visited private services and 2,288 (40.1\%) visited public services. Prevalence of the use of public dental services was greatest among women (41.8\%) and adults with a lower education level (50.2\%). Being non-white $(O R=1.32$, 95\% CI: 1.16, 1.50), lower household income (OR $=2.37,95 \%$ CI: 2.11, 2.65), having had toothache $(O R=1.60,95 \% C I: 1.39,1.83)$, and need for endodontic treatment $(O R=1.44,95 \% C I: 1.12$, 1.85) were associated with public dental service use. Predisposing, enabling, and need factors were associated with public dental care use.
\end{abstract}

Key words Adult, Access to health services, Oral health, Cross-sectional studies
Resumo $O$ objetivo deste artigo é analisar os fatores associados ao uso dos serviços odontológicos públicos por adultos. Estudo transversal com amostra representativa de adultos de 35 a 44 anos, com dados do Levantamento Epidemiológico das Condições de Saúde Bucal da População do Estado de São Paulo (SBSP-2015). Utilizou-se análise de regressão logística multivariada, baseada em modelo proposto por Andersen para predizer o acesso aos serviços odontológicos públicos. Visitaram o serviço privado 3.421 (59,9\%) adultos e 2.288 (40,1\%), o público. Os serviços odontológicos públicos foram mais acessados por mulheres $(41,8 \%)$ e menos escolarizados (50,2\%). Indivíduos não brancos $(O R=1,32 ;$ IC95\%:1,16; 1,50), menor renda familiar $(O R=2,37$; IC95\%:2,11; 2,65), histórico de dor de dente $(O R=1,60 ;$ IC95\%:1,39; 1,83) e necessidade de tratamento endodôntico $(O R=1,44 ;$ IC95\%:1,12; 1,85) foram associados ao uso dos serviços odontológicos públicos. Fatores contextuais, individuais e necessidade de tratamento foram associados à utilização dos serviços odontológicos públicos por adultos residentes no estado de São Paulo, em 2015.

Palavras-chave Adulto, Acesso aos serviços de saúde, Saúde bucal, Estudos transversais de Piracicaba, Universidade Estadual de Campinas. Av. Limeira 901, Areião. 13414900 Piracicaba SP Brasil. sugfonseca@ig.com.br 


\section{Introdução}

O uso dos serviços de saúde representa o núcleo do funcionamento dos sistemas de saúde e estudos recentes mostram desigualdades na utilização dos serviços odontológicos por adultos ${ }^{1-9}$. Nesse contexto, o acesso aos serviços odontológicos é apontado como uma questão complexa e de difícil explicação, principalmente em países como o Brasil que seguem o modelo universal e o acesso aos serviços de saúde é um direito de todos os cidadãos ${ }^{2,3,5-9}$.

No Brasil, estudo com adultos, em 2010, observou prevalência de $75,2 \%$ para necessidade de tratamento dentário e $38,3 \%$ dos entrevistados utilizaram serviço odontológico público ${ }^{10}$. Em Minas Gerais, estudo realizado em 2012 mostrou que $31,8 \%$ dos adultos entrevistados utilizaram o serviço odontológico público ${ }^{11}$.

Para enfrentar as iniquidades no acesso à saúde bucal houve a inclusão de Equipes de Saúde Bucal (ESB) na Estratégia Saúde da Família (ESF) e uma reestruturação da Política Nacional de Saúde Bucal (PNSB) com a proposição de ampliar e descentralizar a rede assistencial através do aumento da oferta de serviços odontológicos públicos não especializados e especializados ${ }^{9,12}$. Entretanto, as iniquidades persistem e estudos indicam que a utilização dos serviços de saúde bucal é resultado da interação de fatores individuais, contextuais, necessidade de tratamento e de organização dos serviços de saúde bucal ${ }^{1-4,8,13-21}$.

Dessa forma, torna-se fundamental analisar o tipo de serviço utilizado pela população adulta, dado que os serviços de saúde devem ser bem estruturados para atender as demandas por atendimento odontológico ${ }^{9,13,15,17,20}$. Estudos sobre o uso de serviços odontológicos podem fornecer conhecimentos importantes para contextualizar a população usuária, compreender as motivações para procura, verificar as condições de saúde bucal, aspectos centrais das políticas públicas e do planejamento em saúde. No entanto, ainda existem questões a serem esclarecidas sobre o uso dos serviços odontológicos públicos por adultos ${ }^{3,9,21,22}$.

Nesse sentido, o objetivo desse estudo foi analisar os fatores associados ao uso dos serviços odontológicos públicos por adultos residentes no Estado de São Paulo.

\section{Métodos}

Foi realizado estudo transversal de base populacional com amostra representativa para a população adulta do estado de São Paulo.
O estado está localizado na região sudeste do Brasil, composto por 645 municípios, o mais populoso estado brasileiro com população estimada para 2016 superior a 44 milhões de habitantes ( $22 \%$ da população brasileira), renda per capta de 1.723 reais, Índice de Desenvolvimento Humano (IDH) de 0,783 (alto), esperança de vida em 2015 de 77,8 anos e $95,9 \%$ da população alfabetizada ${ }^{23}$.

Este estudo utilizou dados do Levantamento Epidemiológico das Condições de Saúde Bucal da População do Estado de São Paulo(SBSP) realizado em 2015. O plano amostral foi elaborado por conglomerados em dois estágios de sorteio com probabilidades proporcionais ao tamanho (PPT) da população, levando em consideração o peso amostral e o efeito de desenhos nas respectivas etapas de sorteio. Este foi um fator de correção nas análises "peso amostral", o qual foi calculado pelo inverso das expressões de probabilidade. O Estado de São Paulo foi estratificado em seis Macrorregiões (Macro) que serão denominados de domínios $^{24}$. Em cada domínio foram sorteados 33 municípios, denominados Unidades Primárias de Amostragem (UPA), com exceção da Macro I (Região Metropolitana da Capital) onde foram sorteados 12 municípios, além da capital ${ }^{24}$. Em seguida, foram sorteados dois Setores Censitários (Unidade Secundária de Amostragem - USA) em cada município sorteado, também respeitando a probabilidade proporcional ao número de habitantes nos setores, enquanto na cidade de São Paulo foram sorteados 36 USA (correspondendo a 18 pontos de coleta). Todos os domicílios do setor sorteado foram percorridos para localizar adultos do grupo etário índice ${ }^{24}$.

Nesse estudo, considerou-se o grupo etário de 35 a 44 anos. A Organização Mundial da Saúde (OMS) recomenda essa faixa etária em inquéritos epidemiológicos porque permite avaliar a saúde bucal e os efeitos gerais do tratamento prestado, permitindo boa aferição das condições de saúde bucal em adultos ${ }^{22,24}$.

A prevalência de cárie dentária foi utilizada como padrão de referência para o cálculo da amostra, conforme já utilizado nos mais recentes levantamentos nacionais e pelo fato da cárie ser o principal problema de saúde bucal ${ }^{10,24}$. Contudo, utilizou-se também a base de cálculo dos dados de Condição Periodontal e Uso e Necessidade de Prótese Dentária, tendo como parâmetros os resultados da Pesquisa Nacional de Saúde Bucal (SB Brasil 2010) para a Região Sudeste ${ }^{10}$. A fórmula para o cálculo do tamanho da amostra de examinados foi ajustada pelo tamanho da população adulta de referência do estado de São Paulo, segundo dados do Sistema de Projeção Populacio- 
nal para os municípios do estado de São Paulo da Fundação $\mathrm{SEADE}^{24}$. O cálculo amostral empregou as prevalências da cárie, condição periodontal, uso e necessidade de prótese, ajustado para o tamanho da população ${ }^{10}$. Assim, 6.051adultos compuseram a amostra ${ }^{24}$.

A coleta de dados empregou a técnica do esgotamento com tamanho mínimo de amostragem, onde todos os domicílios da USA (setor censitário) foram visitados seguindo o trajeto planejado, sendo examinados indivíduos do grupo etário do estudo ${ }^{24}$. Os estágios de sorteio por nível foram acrescentados para sustentar a base probabilística do estudo com sorteio de setores censitários como segundo estágio para, em seguida, proceder-se aos exames nos domicílios. A coleta foi finalizada quando os números mínimos de adultos foram alcançados ${ }^{24}$.

Os indivíduos que nunca visitaram o dentista, não souberam informar ou responderam que consultaram outros serviços odontológicos foram excluídos do estudo ${ }^{9,22}$.

Os dados foram coletados por equipes de Cirurgiões-Dentistas (CD) e Auxiliares de Saúde Bucal (ASB), que foram capacitadas utilizandose a técnica de consenso, calculando-se na rodada final o coeficiente de Kappa, ponderado para cada examinador, grupo etário e agravo estudado, tendo o valor de 0,65 como limite mínimo aceitável ${ }^{24}$.

A variável dependente do presente estudo foi o tipo de serviço odontológico utilizado pela última vez: público ou privado/plano de saúde. A seleção das variáveis independentes foi baseada no modelo teórico de determinação da utilização de serviços de saúde revisitado por Andersen (1995) e empregado em estudos sobre utilização de serviços odontológicos ${ }^{9,17,21,22,25}$. Segundo o modelo, a utilização dos serviços de saúde é resultado da interação de características individuais, contextuais, sistema de saúde e do histórico de utilização dos serviços ${ }^{25}$. O autor pressupõe que os determinantes estão divididos em três grupos: fatores de predisposição (relacionadas ao indivíduo e sociodemográficas), facilitação (renda, características do serviço de saúde) e necessidade (percepção da condição de saúde e necessidades de saúde apresentadas $)^{9,21,22}$. As variáveis de predisposição utilizadas foram: idade (35a 45 anos de idade), sexo (masculino e feminino), escolaridade ( $\leq 9$ anos e $\geq 10$ anos de estudo), cor da pele declarada (branca e não branca: preta, parda, amarela e indígena) e se já teve dor de dente ( $\mathrm{sim}$, não). As variáveis de facilitação foram: renda familiar $(\leq \mathrm{R} \$ 1.500,00$, de $\mathrm{R} \$ 1.501,00$ a $\mathrm{R} \$ 2.500,00$ e $\geq \mathrm{R} \$ 2.501,00$ ), quando consultou o dentista pela última vez (menos de 1 ano, mais de 1 ano), motivo da última consulta (revisão, tratamento/ dor/extração) e satisfação com o tratamento (satisfeito, indiferente/insatisfeito). Como variáveis de necessidade foram admitidas: necessidade de tratamento endodôntico (sim, não), necessidade de prótese superior e inferior ( $\operatorname{sim}$, não) e satisfação com a condição bucal/dentes (satisfeito, indiferente/insatisfeito). As variáveis foram dicotomizadas utilizando como parâmetro a mediana.

Primeiramente, a análise de dados envolveu o cálculo das prevalências e análise bruta por meio do teste do qui-quadrado de Pearson. Por se tratar de um estudo com desenho amostral complexo, o intervalo de confiança de 95\% das prevalências foram calculados com base no peso amostral (ponderação dos dados) a partir da taxa de adensamento por setor censitário, o número de entrevistados / examinados e a taxa de não resposta, sendo estas informações fundamentais para correção das análises, pois geram pesos para ponderação dentro do setor e na macrorregião. Em seguida, as variáveis com valor- $\mathrm{p}<0,20$ foram admitidas para a construção do modelo de regressão logística ajustado. Foram calculados $O d d s$ Ratio (OR) com intervalo de confiança de 95\%. Entretanto, as medidas de OR produzidas por essa técnica podem superestimar as associações e não pode ser descartada a hipótese de que os resultados obtidos estejam superestimados ${ }^{14}$. As variáveis que apresentaram nível de significância menor que 0,05 permaneceram no modelo final explicativo para a utilização de serviços odontológicos. Sexo, renda familiar ou escolaridade são consideradas variáveis de confusão ${ }^{14}$. Nesse sentido, foram construídos três modelos logísticos alternativos: no primeiro, incluiu-se as variáveis escolaridade e sexo, no segundo renda familiar e sexo e no terceiro escolaridade e renda familiar ${ }^{14}$. Optou-se por apresentar o primeiro modelo, porque mostrou maior força de associação com o uso de serviços odontológicos públicos ${ }^{14}$. No entanto, as associações com renda familiar e sexo mantiveram-se estatisticamente significativas no modelo bruto. As análises foram realizadas pelo programa PSPP para Windows versão 0.8 .

O projeto de pesquisa foi aprovado pelo Comitê de Ética em Pesquisa da Faculdade de Odontologia de Piracicaba e seguiu a Resolução número 466, de 12 de dezembro de 2012, do Conselho Nacional de Saúde relativa a pesquisa em seres humanos. Um Termo de Consentimento Livre e Esclarecido (TCLE) foi aplicado e assinado por cada pessoa examinada no estudo ${ }^{24}$. 


\section{Resultados}

Dos 6.051 adultos entrevistados, 5.709 $(94,3 \%)$ fizeram parte desse estudo, 342 (5,65\%) foram excluídos, sendo que 2.288 (40,1\%) visitaram pela última vez o serviço público e 3.421 (59,9\%), o serviço privado ou por plano.

As prevalências dos fatores predisponentes para utilização dos serviços odontológicos são apresentadas na Tabela 1 . Os adultos mais jovens $(51,2 \%)$, do sexo feminino $(68,0 \%)$, com maior escolaridade $(50,5 \%)$, brancos $(62,4 \%)$, relataram não ter tido dor de dente $(68,5 \%)$, consultaram o dentista com maior frequência. Em relação ao serviço público, os indivíduos não brancos $(46,4 \%)$, menos escolarizados $(50,2 \%)$ e com histórico de dor de dente $(51,1 \%)$ recorreram a esse tipo de serviço. A utilização dos serviços particulares ou por plano foi mais prevalente entre os adultos mais velhos $(60,8 \%)$, homens $(63,5 \%)$, mais escolarizados $(69,9 \%)$, brancos $(63,7 \%)$ e que não apresentaram dor de dente $(65,1 \%)$.

A utilização dos serviços odontológicos foi maior em adultos com menor renda familiar $(41,3 \%)$, que visitaram o dentista a menos de um ano $(56,1 \%)$, motivados por tratamento/dor/ extração $(75,0 \%)$ e ficaram satisfeitos com o último tratamento recebido $(86,0 \%)$. Dos adultos que visitaram o serviço público, 1.104 (52,1\%) possuíam renda familiar inferior a $1.500,00$ reais, demoram mais de um ano a visitar o dentista $(41,4 \%)$, motivados por tratamento/dor/extração $(43,1 \%)$ e $40,2 \%$ avaliaram positivamente o tratamento recebido nesse tipo de serviço. Por outro lado, o serviço odontológico particular ou por plano de saúde foi mais utilizado por adultos com maior renda familiar $(77,8 \%)$, que visitaram o dentista a menos tempo $(61,3 \%)$, motivados por revisão $(67,8 \%)$ e foram indiferentes ou insatisfeitos com o último tratamento recebido $(61,2 \%)$ (Tabela 2).

Em relação às variáveis de necessidade, 351 $(6,1 \%)$ dos adultos necessitavam de tratamento endodôntico, 1.835 (32,2\%) algum tipo de prótese superior, $2.654(46,6 \%)$ algum tipo de prótese inferior e $3.132(55,3 \%)$ demonstraram indiferença ou insatisfação com a sua condição bucal/ dental. Entre os adultos que buscaram o serviço público odontológico, 187 (53,3\%) necessitavam de tratamento endodôntico, $887(48,3 \%)$ de algum tipo de prótese superior, $1.220(46,0 \%)$ de prótese inferior e $1.355(43,3 \%)$ relataram indiferença ou insatisfação com a condição bucal ou dos dentes (Tabela 3 ).

Na Tabela 4 está apresentado o modelo final da análise de regressão logística que prediz os fatores associados ao uso de serviço odontológico público por adultos. O modelo contemplou fatores predisponentes, facilitadores e de necessidade associados ao uso de serviço odontológico público. No modelo multivariado, grande parte das variá-

Tabela 1. Prevalência dos fatores predisponentes ao uso dos serviços odontológicos públicos e privados por adultos residentes no estado de São Paulo, Brasil, 2016.

\begin{tabular}{|c|c|c|c|c|c|c|c|c|}
\hline \multirow{2}{*}{ Variáveis } & \multicolumn{3}{|c|}{ Particular/Plano } & \multicolumn{3}{|c|}{ Público } & \multirow{2}{*}{ Total (\%) } & \multirow{2}{*}{ p-valor ${ }^{b}$} \\
\hline & $\mathbf{n}$ & $\%$ & IC $_{95 \%}{ }^{a}$ & $\mathbf{n}$ & $\%$ & IC $_{95 \%}{ }^{a}$ & & \\
\hline Idade & & & & & & & & 0,204 \\
\hline 35 a 39 anos & 1.728 & 59,1 & $(58,4 ; 59,8)$ & 1.195 & 40,9 & $(40,8 ; 50,0)$ & $2.923(51,2)$ & \\
\hline 40 a 45 anos & 1.693 & 60,8 & $(60,0 ; 61,6)$ & 1.093 & 39,2 & $(39,1 ; 39,3)$ & $2.786(48,8)$ & \\
\hline Sexo & & & & & & & & $<0,001$ \\
\hline feminino & 2.259 & 58,2 & $(57,6 ; 58,8)$ & 1.621 & 41,8 & $(41,1 ; 42,5)$ & $3.880(68,0)$ & \\
\hline masculino & 1.162 & 63,5 & $(62,2 ; 64,8)$ & 667 & 36,5 & $(34,8 ; 38,2)$ & $1.829(32,0)$ & \\
\hline Escolaridade em anos & & & & & & & & $<0,001$ \\
\hline$\leq 9$ anos & 1.405 & 49,8 & $(49,7 ; 49,9)$ & 1.418 & 50,2 & $(50,1 ; 50,3)$ & $2.823(49,5)$ & \\
\hline$\geq 10$ anos & 2.016 & 69,9 & $(69,1 ; 70,7)$ & 869 & 30,1 & $(28,9 ; 31,3)$ & $2.885(50,5)$ & \\
\hline Cor da pele & & & & & & & & $<0,001$ \\
\hline branca & 2.270 & 63,7 & $(63,0 ; 64,4)$ & 1.293 & 36,3 & $(36,2 ; 36,4)$ & $3.563(62,4)$ & \\
\hline não branca & 1.151 & 53,6 & $(52,4 ; 54,8)$ & 995 & 46,4 & $(45,1 ; 47,7)$ & $2.146(37,6)$ & \\
\hline Dor de dente & & & & & & & & $<0,001$ \\
\hline $\operatorname{sim}$ & 872 & 48,9 & $(47,3 ; 50,5)$ & 913 & 51,1 & $(49,6 ; 52,6)$ & $1.785(31,5)$ & \\
\hline não & 2.522 & 65,1 & $(64,6 ; 65,6)$ & 1.353 & 34,9 & $(34,2 ; 35,6)$ & $3.875(68,5)$ & \\
\hline
\end{tabular}


Tabela 2. Prevalência fatores facilitadores ao uso de serviços odontológicos públicos e privados por adultos residentes no estado de São Paulo, Brasil, 2016.

\begin{tabular}{|c|c|c|c|c|c|c|c|c|}
\hline \multirow{2}{*}{ Variáveis } & \multicolumn{3}{|c|}{ Particular/Plano } & \multicolumn{3}{|c|}{ Público } & \multirow{2}{*}{ Total (\%) } & \multirow{2}{*}{ p-valor } \\
\hline & $\mathbf{n}$ & $\%$ & $\mathrm{IC}_{95 \%}^{\mathrm{a}}$ & $\mathbf{n}$ & $\%$ & $\mathrm{IC}_{95 \%}^{\mathrm{a}}$ & & \\
\hline Renda familiar em reais & & & & & & & & $<0,001$ \\
\hline$\leq 1,500,00$ & 1.016 & 47,9 & $(46,6 ; 49,2)$ & 1.104 & 52,1 & $(50,9 ; 53,3)$ & $2.120(41,3)$ & \\
\hline$\geq 1,501,00$ e $\leq 2,500,00$ & 1.080 & 61,8 & $(60,4 ; 63,2)$ & 667 & 38,2 & $(37,5 ; 38,9)$ & $1.747(34,0)$ & \\
\hline$\geq 2,501,00$ & 986 & 77,8 & $(76,1 ; 79,5)$ & 282 & 22,2 & $(19,0 ; 25,4)$ & $1.268(24,7)$ & \\
\hline Última visita ao dentista & & & & & & & & 0,035 \\
\hline menos de um ano & 1.940 & 61,3 & $(60,6 ; 62,0)$ & 1.222 & 38,7 & $(38,6 ; 38,8)$ & $3.162(56,1)$ & \\
\hline mais de um ano & 1.448 & 58,6 & $(58,5 ; 58,7)$ & 1.024 & 41,4 & $(40,2 ; 42,6)$ & $2.472(43,9)$ & \\
\hline Motivo & & & & & & & & $<0,001$ \\
\hline revisão & 938 & 67,8 & $(66,2 ; 69,4)$ & 446 & 32,2 & $(30,2 ; 34,2)$ & $1.384(25,0)$ & \\
\hline tratamento/dor/extração & 2.358 & 56,9 & $(56,4 ; 57,4)$ & 1.789 & 43,1 & $(42,5 ; 43,7)$ & $4.147(75,0)$ & \\
\hline Satisfação com tratamento & & & & & & & & 0,434 \\
\hline satisfeito & 2.916 & 59,8 & $(59,3 ; 60,3)$ & 1.963 & 40,2 & $(39,6 ; 40,8)$ & $4.879(86,0)$ & \\
\hline indiferente/insatisfeito & 488 & 61,2 & $(58,8 ; 63,6)$ & 309 & 38,8 & $(35,6 ; 42,0)$ & $797(14,0)$ & \\
\hline
\end{tabular}

Tabela 3. Prevalência dos fatores de necessidade relacionados ao uso dos serviços odontológicos públicos e privados por adultos residentes no estado de São Paulo, Brasil, 2016.

\begin{tabular}{|c|c|c|c|c|c|c|c|c|}
\hline \multirow{2}{*}{ Variáveis } & \multicolumn{3}{|c|}{ Particular/Plano } & \multicolumn{3}{|c|}{ Público } & \multirow{2}{*}{ Total (\%) } & \multirow{2}{*}{ p-valor } \\
\hline & $\mathbf{n}$ & $\%$ & $\mathrm{IC}_{95 \%}{ }^{\mathrm{a}}$ & $\mathbf{n}$ & $\%$ & IC $_{95 \%}{ }^{a}$ & & \\
\hline Endodontia & & & & & & & & $<0,001$ \\
\hline $\operatorname{sim}$ & 164 & 46,7 & $(46,0 ; 47,4)$ & 187 & 53,3 & $(46,9 ; 59,7)$ & $351(6,1)$ & \\
\hline não & 3.257 & 60,8 & $(60,4 ; 61,2)$ & 2.101 & 39,2 & $(38,6 ; 39,8)$ & $5.358(93,2)$ & \\
\hline Prótese superior & & & & & & & & $<0,001$ \\
\hline $\operatorname{sim}$ & 948 & 51,7 & $(50,3 ; 53,1)$ & 887 & 48,3 & $(46,9 ; 49,7)$ & $1.835(32,2)$ & \\
\hline não & 2.463 & 63,8 & $(63,3 ; 64,2)$ & 1.398 & 36,2 & $(35,5 ; 36,9)$ & $3.861(67,8)$ & \\
\hline Prótese inferior & & & & & & & & $<0,001$ \\
\hline $\operatorname{sim}$ & 1.434 & 54,0 & $(53,0 ; 55,0)$ & 1.220 & 46,0 & $(44,9 ; 47,1)$ & $2.654(46,6)$ & \\
\hline não & 1.977 & 65,0 & $(64,3 ; 65,7)$ & 1.064 & 35,0 & $(34,0 ; 36,0)$ & $3.041(53,4)$ & \\
\hline Satisfação com a boca/dente & & & & & & & & $<0,001$ \\
\hline satisfeito & 1.624 & 64,1 & $(63,1 ; 65,1)$ & 909 & 35,9 & $(34,6 ; 37,2)$ & $2.533(44,7)$ & \\
\hline indiferente/insatisfeito & 1.777 & 56,7 & $(55,9 ; 57,5)$ & 1.355 & 43,3 & $(42,3 ; 44,3)$ & $3.132(55,3)$ & \\
\hline
\end{tabular}

veis predisponentes e de facilitação permaneceram fortemente associadas ao desfecho. Dentre os fatores predisponentes: mulheres, menor escolaridade, não brancos e com histórico de dor de dente se mantiveram associados ao uso de serviço odontológico público. A visita ao serviço público foi considerado um fator protetivo para o sexo masculino $\left(\mathrm{OR}=0,85 ; \mathrm{IC}_{95 \%}: 0,74 ; 0,96\right)$ e com escolaridade igual ou superior a dez anos $\left(\mathrm{OR}=0,52 ; \mathrm{IC}_{95 \%}: 0,46\right.$; $0,60)$. Os indivíduos com histórico de dor de den- te possuíam $1,6\left(\mathrm{IC}_{95 \%:} 1,39 ; 1,83\right)$ mais chances de procurarem o serviço público quando comparados com os indivíduos que não possuíam histórico de dor de dente. Os adultos com menor renda familiar possuíam 2,37 $\left(\mathrm{IC}_{95 \%}: 2,11 ; 2,65\right)$ mais chances de procurarem o serviço público quando comparados com os indivíduos com maior renda. A necessidade de tratamento endodôntico foi associada ao uso de serviço odontológico público por adultos $\left(\mathrm{OR}=1,44 ; \mathrm{IC}_{95 \%}: 1,12 ; 1,85\right)$. A retirada da variável 
Tabela 4. Fatores predisponentes, facilitadores e de necessidade associados ao uso dos serviços odontológicos públicos por adultos do estado de São Paulo, Brasil, 2016.

\begin{tabular}{|c|c|c|c|c|}
\hline Variáveis & $\mathrm{OR}^{\mathrm{a}}$ & IC $_{95 \%}{ }^{\mathrm{b}}$ & p-valor ${ }^{\mathrm{c}}$ & p-valor ${ }^{d}$ \\
\hline \multicolumn{5}{|l|}{ Predisponentes } \\
\hline Sexo & & & 0,012 & 0,002 \\
\hline feminino & 1 & & & 1 \\
\hline masculino & 0,85 & $(0,74 ; 0,96)$ & & $1,21(1,07 ; 1,37)$ \\
\hline Escolaridade em anos & & & $<0,001$ & $<0,001$ \\
\hline$\leq 9$ anos & 1 & & & 1 \\
\hline$\geq 10$ anos & 0,52 & $(0,46 ; 0,60)$ & & $0,47(0,42 ; 0,53)$ \\
\hline Cor da pele & & & $<0,001$ & $<0,001$ \\
\hline branca & 1 & & & 1 \\
\hline não branca & 1,32 & $(1,16 ; 1,50)$ & & $1,36(1,21 ; 1,53)$ \\
\hline Dor de dente & & & $<0,001$ & $<0,001$ \\
\hline não & 1 & & & 1 \\
\hline $\operatorname{sim}$ & 1,60 & $(1,39 ; 1,83)$ & & $1,64(1,45 ; 1,86)$ \\
\hline \multicolumn{5}{|l|}{ Facilitadores } \\
\hline Renda familiar & & & $<0,001$ & - \\
\hline$\geq \mathrm{R} \$ 1,500,00$ & 1 & & & - \\
\hline$\leq \mathrm{R} \$ 1,501,00$ & 2,37 & $(2,11 ; 2,65)$ & & - \\
\hline \multicolumn{5}{|l|}{ Necessidade } \\
\hline Endodontia & & & 0,005 & 0,003 \\
\hline não & 1 & & & 1 \\
\hline $\operatorname{sim}$ & 1,44 & $(1,12 ; 1,85)$ & & $1,41(1,12 ; 1,78)$ \\
\hline R2 de Nagelkerke & 0,13 & & & \\
\hline
\end{tabular}

a) OR (odds ratio: razão de chances). b) $\mathrm{IC}_{95 \%}$ (Intervalo de Confiança de $95 \%$ ). c) p-valor: probabilidade de significância pelo teste de Wald. d) p-valor: probabilidade de significância pelo teste de Wald - modelo sem renda familiar.

"renda familiar" do modelo alterou as variâncias das estimativas do modelo bruto, principalmente, para a variável sexo. No modelo ajustado, os homens possuíam 1,21 mais chances de procurarem o serviço odontológico público quando comparados com as mulheres. No entanto, o modelo sem a variável renda familiar manteve a significância estatística com as demais variáveis com ganhos para as variáveis sexo e necessidade de tratamento endodôntico.

\section{Discussão}

Nesse estudo, a prevalência de uso dos serviços odontológicos públicos por adultos foi próxima de $40 \%$ e esse achado foi superior ao encontrado em 2012 em Minas Gerais ${ }^{1}$. No Maranhão (MA), estudo com dados de 2007, em municípios com mais de 100 mil habitantes mostrou que a natureza do atendimento por plano ou particular obteve prevalência de 55,6\% em adultos ${ }^{17}$. Estudo com dados nacionais realizado em 2003 mostrou que 51,8\% dos adultos visitaram pela última vez o serviço odontológico público ${ }^{22}$. Entretanto, as divergências encontradas para as prevalências do uso de serviços odontológicos podem ser explicada pelas diferenças na faixas etárias empregadas para caracterizar a população adulta ${ }^{11,17,22}$.

Segundo Andersen, os fatores predisponentes e facilitadores relacionados ao indivíduo e família devem perceber a ocorrência de doenças para que ocorra a procura por serviços odontológicos ou médicos ${ }^{25}$. Entre as variáveis predisponentes e facilitadoras, as mulheres, menos escolarizados, indivíduos não brancos e com menor renda familiar utilizaram mais os serviços públicos odontológi$\cos ^{9}$. A maior utilização de serviços odontológicos públicos pelas mulheres pode estar relacionada a uma maior disponibilidade de tempo para comparecer às unidades de saúde pública no horário de funcionamento ${ }^{22}$.

No modelo multivariável, a escolaridade (predisposição) e a renda familiar (facilitação) mostraram-se associadas ao desfecho. A renda familiar traduz melhores condições de renda, empregabilidade e poder de compra por serviço odontológico pelo sistema privado e/ou de con- 
vênios enquanto escolaridade representa o nível de entendimento ou consciência sobre as doenças e cuidados bucais ${ }^{1,3-5,9,21,22}$. Ademais, para Andersen ${ }^{15}$ e outros autores ${ }^{25}$, as diferenças na condição socioeconômica (renda familiar) indicam presença de iniquidade (desigualdade injusta) no uso dos serviços odontológicos.

A variável dor de dente (predisponente) e as variáveis de necessidade de tratamento associadas ao uso de serviços odontológicos públicos podem fornecer reflexões importantes sobre os impactos da política de saúde bucal sobre a saúde bucal coletiva e qualidade de vida ${ }^{12,26}$. Nesse sentido, confirmam o perfil epidemiológico de elevadas prevalências de problemas bucais e de perdas dentárias em adultos ${ }^{10,27}$. A inserção da ESB na ESF, a partir de 2000, e a reestruturação da PNSB em 2004 trouxeram avanços a partir de incentivos financeiros em todos os níveis de atenção como: Implantação de Equipes de Saúde Bucal; Implantação dos Centros de Especialidade Odontológica (CEO), Laboratórios Regionais de Prótese Dentária (LRPD) ${ }^{12,26,28}$. Como efeito permitiram a descentralização das ações de saúde bucal e impulsionaram o acesso da população aos serviços odontológicos públicos não especializados e especializados ${ }^{12,26,28}$. A melhora da renda média da população brasileira e da taxa de emprego (empregabilidade) podem ter influenciado o aumento da procura de serviço odontológico pelo sistema privado e/ou de convênios. Por outro lado, as altas taxas de desemprego atuais podem ter motivado os adultos a buscarem o serviço público como alternativa de acesso ao serviço odontológico, ou seja, existem fortes evidências da necessidade de manutenção e fortalecimento da PNSB como política indutora da inclusão social e equidade.

Entretanto, não está claro se o aumento da oferta de serviços odontológicos especializados, não especializados e a descentralização da PNSB foram capazes de reduzir as desigualdades no acesso à saúde bucal ${ }^{27,28}$. A Pesquisa Nacional de Saúde (PNS), que analisou o acesso e utilização de brasileiros com mais de 18 anos aos serviços odontológicos, identificou que as unidades básicas de saúde responderam por $19,6 \%$ dos atendimentos odontológicos e $11,0 \%$ perderam todos os dentes, o que corresponde a um contingente de 16 milhões de dentes perdidos por brasilei$\operatorname{ros}^{29}$. Ademais, estudos anteriores verificaram menores prevalências de utilização dos serviços odontológicos para revisão ou prevenção no serviço odontológico público ${ }^{9,10}$. Em Minas Gerais, estudo com dados de 2012 identificou que apenas
22,6\% dos adultos visitaram o dentista motivados por prevenção ou revisão9 ${ }^{9}$ Nesse sentido, estudos sugerem que a inclusão da ESB na unidade de saúde da família não se traduziu em mudança do processo de trabalho tradicional e sugere que essa situação seja consequência da histórica desassistência odontológica à população adulta e que as ações preventivas são prioritárias para crianças e não atingem os adultos ${ }^{22,26-28}$.

O nível de necessidade indica a causa mais direta de utilização dos serviços odontológicos ${ }^{27}$. Nesse sentido, a necessidade de tratamento endodôntico foi associada ao tipo de serviço odontológico na análise ajustada. Esse achado evidencia que os usuários de serviços públicos demandaram tratamentos curativos e reabilitadores, de maior complexidade e que apresentavam piores condições de saúde bucal ${ }^{22}$. Também sugere a existência de demanda reprimida por esse tipo de serviço para a população estudada e evidencia que a população menos favorecida socioeconomicamente tenha acesso a serviços odontológicos especializados, o que foi também detectado em estudos internacionais ${ }^{3-5}$ e brasileiros ${ }^{9,15,18,27}$. Esse fato pode ter relação com a forma de organização do acesso aos serviços odontológicos públicos no Brasil, que priorizava o atendimento materno-infantil, escolares e indivíduos com maior necessidade de tratamento no primeiro momento de organização da atenção ${ }^{26,28}$. A lógica de assistência odontológica voltada para a resolução de problemas bucais é contraditória ao preconizado pela moderna atenção odontológica, centrada na vigilância e monitoramento da saúde bucal, nas ações preventivas, com baixa frequência de restaurações e extrações ${ }^{5,22}$. No entanto, algumas variáveis de necessidade e de predisposição (dor de dente) possuem potencial de modificação por profissionais de saúde através de ações preventivas, empoderamento de sujeitos para mudar comportamentos, crenças, atitudes e de valoração que os indivíduos atribuem à saúde bucal ${ }^{21}$.

Em razão do desenho de estudo ser transversal, os resultados obtidos de associação não permitem estabelecer causalidade e buscou-se identificar marcadores individuais do tipo de serviço odontológico utilizado por adultos. Embora a recusa em participar do estudo tenha sido baixa, o horário da coleta pode ter impossibilitado a participação de indivíduos por estarem ausentes dos domicílios ou no trabalho. Esse fato implicou em expressiva participação de mulheres na amostra. Assim, a maior utilização de serviços odontológicos por mulheres pode ter sido superestimada e comprometer a validade externa do estudo. $\mathrm{Na}$ 
mesma direção, a técnica estatística de OR pode influenciar a variância das estimativas indicando cautela na interpretação dos resultados ${ }^{30}$. Por outro lado, o estudo possui abrangência, riqueza de dados provenientes de exames clínicos e critérios metodológicos robustos que lhe conferem validade interna. Apesar da baixa taxa de não resposta e da exclusão de adultos que relataram nunca terem visitado o dentista e responderam que consultaram outros tipos de serviços odontológicos podem representar um padrão diferente de utilização dos serviços odontológicos. Por fim, algumas variáveis utilizadas dependiam, da memória dos entrevistados e pode ter ocorrido viés de resposta. Alguns indivíduos podem ter relatado maior procura por tratamento odontológico, consultas mais recentes ao dentista, para não parecerem negligentes.

\section{Colaboradores}

SGO Fonseca e MC Meneghim participaram da concepção, delineamento e redação do artigo. EP Fonseca colaborou com a análise e interpretação dos dados. MC Meneghim realizou a revisão crítica do artigo. Todos os autores aprovaram a versão a ser publicada.
Em síntese, o estudo mostrou desigualdades no acesso aos serviços odontológicos por adultos no estado de São Paulo. As prevalências indicaram que os serviços privados/por plano de saúde foram mais utilizados que os serviços públicos odontológicos. A utilização do modelo teórico proposto por Andersen permitiu identificar fatores predisponentes, facilitadores e de necessidade capazes de explicar os fatores moduladores do uso dos serviços odontológicos públicos por adultos. As variáveis associadas: escolaridade, dor de dente e necessidade de tratamento endodôntico na utilização do serviço odontológico sugerem o aprimoramento de mecanismos de acesso, principalmente os ligados à informação. A expectativa é que as evidências encontradas possam induzir mudanças positivas no contexto da atenção à saúde bucal de adultos brasileiros, independentemente do tipo de serviço utilizado. 


\section{Referências}

1. Gilbert HG, Duncan RP, Bruce V. Determinants of dental care use in dentate adults: six monthly use during a 24-month period in the Florida Dental Care Study. Soc Sci Med 1998; 47(6):727-737.

2. Tennstedt SL, Brambilla DL, Jette AM, McGuire SM. Understanding dental service use by older adults sociobehavioral factors vs. need. J Public Health Dent 1994; 54(4):211-219.

3. Nguyen L, Häkkinen U. Choices and utilization in dental care Public vs. private dental sectors, and the impact of a two-channel financed health care system. Eur J Health Econ 2006; 7(2):99-106.

4. Luzzi L, Spencer AJ. Public dental service utilization among adults in South Australia. Aust Dent J 2009; 54(2):154-160.

5. Brennan DS, Luzzi L, Roberts-Thomson KF. Dental service patterns among private and public adult patients in Australia. BMC Health Serv Res 2008; 3:1-8.

6. Hancock M, Calnan M, Manley G. Private or NHS General Dental Service care in the United Kingdom? A study of public perception and experience. J Public Health Med 1999 Dec; 21(4):415-20.

7. Ajayi DM, Arigbede AO. Barriers to oral health care utilization in Ibadan, South West Nigeria. Afr Health Sci 2012; 12(4):507-513.

8. Locker D, Maggirias J, Quiñonez C. Income, dental insurance coverage, and financial barriers to dental care among Canadian adults. J Public Health Dent 2011; 71(4):327-334.

9. Pinto RS, Abreu MHNG, Vargas AMD. Comparing adult users of public and private dental services in the state of Minas Gerais, Brazil. BMC Oral Health 2014; 14:100.

10. Brasil. Ministério da Saúde (MS). SB Brasil 2010: pesquisa Nacional de Saúde Bucal: Resultados Principais. Brasília: MS; 2012.

11. Minas Gerais. Secretaria de Estado de Saúde (SES). Pesquisa das condições de saúde bucal da população mineira - Resultados principais. Belo Horizonte: Editora Autêntica; 2013.

12. Pucca Jr GA, Gabriel M, Araújo ME, Almeida FCS. Ten Years of a National Oral Health Policy in Brazil: Innovation, Boldness, and Numerous Challenges. $J$ Dent Res 2015; 94(10):1333-1337.

13. Manhães ALD, Costa AJL. Acesso a e utilização de serviços odontológicos no Estado do Rio de Janeiro, Brasil, em 1998: um estudo exploratório a partir da Pesquisa Nacional por Amostra de Domicílios. Cad Saude Publica 2008; 24(1):207-218.

14. Matos DL, Lima-Costa MFF, Guerra HL, Marcenes W. Projeto Bambuí: estudo de base populacional dos fatores associados com o uso regular de serviços odontológicos em adultos. Cad Saude Publica 2001; 17(3):661-668.

15. Peres KG, Peres MA, Boing AF, Bertoldi AD, Bastos JL, Barros AJD. Redução das desigualdades sociais na utilização de serviços odontológicos no Brasil entre 1998 e 2008. Rev Saude Publica 2012; 46(2):250-258.

16. Pinheiro RS, Torres TZG. Uso de serviços odontológi- cos entre os Estados do Brasil. Cien Saude Colet 2006; 11(4):999-1010.

17. Gomes AMM, Thomaz EBAF, Alves MTSSB, Silva AAM, Silva RA. Fatores associados ao uso dos serviços de saúde bucal: estudo de base populacional em municípios do Maranhão. Brasil. Cien Saude Colet 2014; 19(2):629-640.

18. Baldani MH, Antunes JLF. Inequalities in access and utilization of dental services: a cross-sectional study in an area covered by the Family Health Strategy. Cad Saude Publica 2011; 27(Supl. 2):272-283.

19. Pereira CRS, Patrício AAR, Araújo FAC, Lucena EES, Lima KC, Roncalli AG. Impacto da Estratégia Saúde da Família com equipe de saúde bucal sobre a utilização de serviços odontológicos. Cad Saude Publica 2009; 25(5):985-996.

20. Araújo CS, Lima RC, Peres MA, Barros AJD. Utilização de serviços odontológicos e fatores associados: um estudo de base populacional no Sul do Brasil. Cad Saude Publica 2009; 25(5):1063-1072.

21. Camargo MBJ, Dumith SC, Barros AJD. Uso regular de serviços odontológicos entre adultos: padrões de utilização e tipos de serviço. Cad Saude Publica 2009; 25(9):1894-1906.

22. Pinto RS, Matos DL, Loyola Filho AI. Characteristics associated with the use of dental services by the adult Brazilian population. Cien Saude Colet 2012 Fev; 17(2):531-544.

23. Instituto Brasileiro de Geografia e Estatística (IBGE). Censo Demográfico 2010. Estimativa população para o ano de 2016 e dados sócio-demográficos do Estado de São Paulo [Internet]. Rio de Janeiro: IBGE; 2016 [citado 2017 Maio 18]. Disponível em: http://ibge.gov.br/ estadosat/perfil.php?sigla $=$ sp

24. Pereira AC, Frias AC, Vieira V. Pesquisa Estadual de Saúde Bucal. - SB São Paulo 2015. Águas de São Pedro: Livronovo; 2016.

25. Andersen RM. Revisiting the behavioral model and access to medical care: does it matter? J Health Soc Behav 1995,36(1):1-10.

26. Antunes JLF, Narvai PC. Políticas de saúde bucal no Brasil e seus impactos sobre as desigualdades em saúde. Rev Saude Publica 2010; 44(2):360-365.

27. Baldani MH, Brito WH, Lawder JAC, Mendes YBE, Silva FFM, Antunes JLF. Determinantes individuais da utilização de serviços odontológicos por adultos e idosos de baixa renda. Rev Bras Epidemiol 2010; 13(1):150-162.

28. Soares CLM, Paim JS. Aspectos críticos para a implementação da política de saúde bucal no Município de Salvador, Bahia, Brasil. Cad Saude Publica 2011; 27(5):966-974.

29. Instituto Brasileiro de Geografia e Estatística (IBGE). Pesquisa Nacional de Saúde: 2013: acesso e utilização dos serviços de saúde, acidentes e violências: Brasil, grandes regiões e unidades da federação [Internet]. Rio de Janeiro: IBGE; 2015. [citado 2017 Jan 10]. Disponível em: http://biblioteca.ibge.gov.br/visualizacao/ livros/liv94074.pdf 
30. Francisco PMSB, Donalisio MR, Barros MBA, Cesar CLG, Carandina L, Goldbaum M. Medidas de associação em estudo transversal com delineamento complexo: razão de chances e razão de prevalência. Rev Bras Epidemiol 2008; 11(3):347-355.

Artigo apresentado em 14/09/2017

Aprovado em 16/05/2018

Versão final apresentada em 18/05/2018 